\title{
El consumo de la polidextrosa previene la obesidad y sus comorbilidades en ratas alimentados con dieta hipercalórica
}

\section{Polydextrose consumption prevents obesity and comorbidities in rats fed with a hypercaloric diet}

\begin{abstract}
RESUMEN
El objetivo de este trabajo fue evaluar el efecto de las diferentes concentraciones de polidextrosa en la prevención de la obesidad y sus comorbilidades, en ratas alimentados con dieta hipercalórica. Se utilizaron ratas machos Wistar, repartidos en 4 grupos: Grupo control (HC) y 3 grupos que recibieron dieta hipercalórica con suplementación del $2 \%$, $4 \%$ y $6 \%$ de polidextrosa (HC2\%P, HC4\%P y HC6\%P respectivamente). La dieta hipercalórica utilizada fue la del tipo de cafetería para inducir la obesidad. Se midió peso corporal e ingesta de la dieta, se realizaron pruebas de tolerancia a la glucosa y a la insulina. Los animales fueron sometidos a eutanasia para toma de muestra de sangre medidas antropométricas y pesaje de órganos y tejidos. La polidextrosa disminuyó significantemente el peso, la grasa corporal, la glicemia, los triglicéridos, la intolerancia a la glucosa y la resistencia a la insulina, y aumentó los niveles del colesterol HDL. Se concluye que el consumo de polidextrosa redujo las complicaciones derivadas de la obesidad en ratas alimentados con dieta hipercalórica, siendo éste un potencial tratamiento para el control de la obesidad, la diabetes tipo Il y las enfermedades cardiovasculares.

Palabras clave: Diabetes; Obesidad; Prebiótico; Polidextrosa; Ratas.
\end{abstract}

\footnotetext{
ABSTRACT

The aim of this study was to evaluate the effect of different polydextrose concentrations for the prevention of obesity and its comorbidities in rats fed a high calorie diet. Thirty male Wistar rats were used. Rats were divided into 4 groups: Control group (HC) and 3 groups which received a hypercaloric diet with $2 \%, 4 \%$ and $6 \%$ polydextrose supplementation (HC2\%P, HC4\%P and HC6\%P, respectively). The hypercaloric diet used was of the cafeteria type to induce obesity. Body weight and feed intake were verified weekly. Glucose and insulin tolerance tests were performed five days before finalizing the experiment. At the end of the experiment, animals were euthanized for blood collection, anthropometric measurements and tissue weighing. Polydextrose significantly decreased weight, body fat, blood glucose, triglycerides, glucose intolerance and
}

Suene Vanessa da Silva Souza ${ }^{1}$, Camila Jordão $C^{1}$, Danieli Zampieri', Bruna Spontoni do Espírito $S^{1}$, Julia Leite $W^{1}$, Adriana Conceicon Guercio' ${ }^{2}$, Wander de Oliveira $F^{1}$, Elisvânia Freitas dos $S^{1^{*}}$.

1. Facultad de Ciencias Farmacéuticas, Alimentación y Nutrición (FACFAN), Universidad Federal de Mato Grosso do Sul (UFMS), Campo Grande, Mato Grosso do Sul, Brasil.

2. Médica Veterinaria. Bioterio Central de la Universidad Federal de Mato Grosso do Sul (UFMS), Campo Grande, Mato Grosso do Sul, Brasil.

*Dirigir correspondência a: Elisvânia Freitas dos Santos. Universidad Federal de Mato Grosso do Sul (UFMS), Campo Grande, MS, Brasil. Avenida Costa e Silva, s/n. Cidade Universitária, Campo Grande - MS, 79070-900. Teléfono móvil:+556781155325 E-mail: elisvania@gmail.com

insulin resistance and increased HDL cholesterol levels. The use of polydextrose reduced the complications of obesity in mice fed a hypercaloric diet. In conclusion, polydextrose may be a promising treatment for controlling obesity, diabetes type II and cardiovascular diseases.

Keywords: Diabetes; Obesity; Polydextrose; Prebiotics; Rats.

\section{INTRODUCCIÓN}

En los últimos tiempos, las transiciones epidemiológicas, nutricionales y demográficas han revelado un aumento de las muertes y morbilidades por Enfermedades Crónicas No Transmisibles (ECNT), siendo la obesidad una de las causas $^{1,2}$. La Organización Mundial de Salud indica que la prevalencia de esa enfermedad ha aumentado en todo el 
mundo. Ella es caracterizada por la acumulación excesiva de grasa corporal, y está asociada a enfermedades crónicas como la diabetes tipo II, la hipertensión y las enfermedades del corazón, dislipidemias, entre otros ${ }^{3}$. Entre las causas de obesidad están varios factores, como: psicológicos, genéticos, metabólicos y ambientales. Este último, que incluye los hábitos alimentarios, ha sido considerado una de las principales causas de la prevalencia de la enfermedad ${ }^{3}$.

Se puede evitar la obesidad a través de varias acciones, como un cambio de los hábitos alimentarios ${ }^{4}$. En base a esto, investigaciones sobre alimentos han ganado un espacio cada vez mayor. Entre esos estudios están los prebióticos, que son ingredientes alimentarios no digeribles por el organismo, pero que promueven el crecimiento de bacterias beneficiosas importantes para la salud, como las bifidobacterias del colon ${ }^{5}$. Estas bacterias aportan beneficios como la disminución de las enfermedades intestinales y cardiovasculares, la diabetes y la obesidad ${ }^{5,6}$.

Entre las varias sustancias prebióticas existentes, como la inulina, los fructooligosacáridos (FOS), los galactooligosacáridos (GOS), se puede mencionar la polidextrosa, un polisacárido sintetizado por la polimerización de la glucosa, de bajo valor calórico (1 $\mathrm{kcal} / \mathrm{g}$ ), parcialmente fermentable y de baja viscosidad ${ }^{5,7}$. Asimismo, ese prebiótico puede considerarse como una fibra soluble, por contener características similares ${ }^{7}$. Según estudios, la polidextrosa presenta funciones como el aumento de la saciedad, la oxidación de sustratos y la mejora de las condiciones de la microbiota intestinal, que contribuyen a la reducción de los niveles de glucosa, lípidos séricos y enfermedades crónicas ${ }^{8,9}$. Una investigación en humanos mostró que ese prebiótico favoreció la función intestinal e inhibió la absorción excesiva de glucosa, contribuyendo a la mejora de las funciones fisiológicas ${ }^{7}$. De este modo, el objetivo de este trabajo fue el de evaluar el efecto de las diferentes concentraciones de polidextrosa en la prevención de la obesidad y sus comorbilidades, en ratas alimentados con dieta hipercalórica.

\section{MATERIALES Y MÉTODOS Animales}

Para el desarrollo de esta investigación se utilizaron 30 ratas machos del linaje Wistar, de 21 días de edad, recién destetados (76 $\pm 9 \mathrm{~g})$, suministrados por el Bioterio Central de la Universidad Federal de Mato Grosso do Sul (UFMS), Campo Grande - MS. Las ratas fueron puestas en jaulas colectivas en el laboratorio de Ensayos Biológicos, en el que se controlaron las condiciones ambientales a fin de mantener la temperatura a $22 \pm 2{ }^{\circ} \mathrm{C}$, humedad relativa del aire en un 50-60\% y ciclo luz/oscuridad de 12 horas.

El trabajo fue aprobado por la Comisión de Ética en el Uso de Animales/CEUA/UFMS (Protocolo n ${ }^{\circ}$ 607/2014). Todos los procedimientos se realizaron según las normas recomendadas por el Consejo Nacional de Control de Experimentación Animal (CONSEA).

\section{Diseño experimental y dietas}

Se repartieron los animales experimentales en dos grupos:

1) Grupo Control con Dieta Hipercalórica ( $\mathrm{HC}-4,5$ $\mathrm{kcal} / \mathrm{g}$ ) $\mathrm{n}=6$;

2) Grupos experimentales con dieta hipercalórica suplementada con polidextrosa, siendo:

a) Dieta Hipercalórica con suplementación del 2,0\% de polidextrosa $(\mathrm{HC} \% \mathrm{P}-4,5 \mathrm{kcal} / \mathrm{g}) \mathrm{n}=8$;

b) Dieta Hipercalórica con suplementación del 4,0\% de polidextrosa $(\mathrm{HC} 4 \% \mathrm{P}-4,5 \mathrm{kcal} / \mathrm{g}) \mathrm{n}=8$; c) Dieta Hipercalórica con suplementación del 6,0\% de polidextrosa $(\mathrm{HC} 6 \% \mathrm{P}-4,4 \mathrm{kcal} / \mathrm{g}) \mathrm{n}=8$.

La dieta hipercalórica utilizada en este experimento fue un tipo de dieta de cafetería para inducir la obesidad, previamente estandarizada y adaptada de Estadella et al. ${ }^{10}$. Fueron mezclados alimentos hipercalóricos: la ración comercial, cacahuete tostado, achocolatado en polvo y galletas del tipo wafer sabor chocolate, en la proporción de 3:2:2:1. La composición de las dietas del experimento se describe en la tabla 1.

Los animales recibieron dieta y agua en condiciones ad libitum por 8 semanas (60 días - periodo experimental). Las dietas fueron sustituidas tres veces a la semana para el control de la ingesta alimentaria, considerando la diferencia, en gramos, entre la cantidad suministrada y la cantidad restante. $Y$ en dicho periodo los animales fueron sometidos a pesaje para control de la ganancia de peso. Se calcularon la Ingesta de Energía (IE) y la Eficiencia Alimentaria (EA) según Novelli et al. ${ }^{11}$.

IE $($ Kcal/día $)=$ consumo alimentario promedio

$x$ valor energético

EA $(\%)=$ promedio de ganancia de peso

corporal x 100/ingesta de energía

Cinco días antes de concluir el experimento, los animales se quedaron en ayuno por seis horas, para la realización de la prueba de tolerancia a la glucosa (PTG), a través de la que se verificó la glicemia de ayuno vía caudal (tiempo 0), con el uso de un glucómetro (INJEX ${ }^{\circledR}$ Sens II). Posteriormente, los animales recibieron, por alimentación forzada, glucosa en la concentración de $1 \mathrm{~g} / \mathrm{Kg}$ de peso corporal. La glicemia fue verificada a los 20, 40, 60 y 120 minutos tras la administración de glucosa, adaptado de Lenquiste et al. ${ }^{12}$. Tras la prueba, los animales volvieron a recibir las dietas normalmente. Los resultados fueron usados para calcular el área bajo la curva (AUC) del gráfico de glucosa en función del tiempo. Al día siguiente, para realización de la prueba de tolerancia a insulina (PTI), los animales fueron sometidos, nuevamente, al ayuno por 6 horas. Se verificó, además, la glicemia de ayuno vía caudal (tiempo 0). La prueba fue realizada tras una inyección intraperitoneal de $1,5 \mathrm{UI} / \mathrm{Kg}$ de insulina $\left(\right.$ NovoRapid $^{\circledR}$ FlexPen $\left.{ }^{\circledR}\right)$, la glicemia fue verificada en los tiempos 15, 30, 45 y 60 min, adaptado Lenquiste et al. ${ }^{12}$. Se utilizaron los resultados para calcular la constante de reducción de la glucosa (kITT). Para el 
cálculo del kITT se utilizó la regresión lineal (tiempo x Ln [glucosa]) multiplicada por 100 para obtener la tasa de descomposición de la glucosa constante durante la prueba de tolerancia a insulina (kITT, \%min). Además de ello, las mediciones de glucosa se convirtieron en valores logarítmicos naturales $(\mathrm{Ln})^{13}$.

Tras esas pruebas, los animales recibieron nuevamente la dieta normal. Al final del periodo experimental, se sometieron todos los animales a un ayuno de ocho horas y fueron anestesiados con Ketamina ( $80 \mathrm{mg} / \mathrm{kg}$ de peso) y Xilacina (10 $\mathrm{mg} / \mathrm{kg}$ de peso), para realización de la colecta de sangre por punción cardíaca. A continuación, se sacrificaron los animales en cámara de $\mathrm{CO}_{2}$. Todas las medidas, retiradas de órganos y los tejidos adiposos fueron realizadas en el animal ya muerto.

Las medidas antropométricas tomadas fueron: el largo (naso-anal), midiéndose la distancia entre el hocico y la base de la cola; la Circunferencia Torácica (CT), medida justo detrás de la pata delantera, y la Circunferencia Abdominal (CA), tomada en la parte anterior al antepié ${ }^{11}$. Se realizaron, conforme a la metodología también descrita por Novelli et al. ${ }^{11}$, el Índice de Lee y el Índice de Masa Corporal.

Índice de Lee $(\mathrm{g} 3 / \mathrm{cm})=$ Raíz cúbica de peso corporal

(g) / largo naso-anal $(\mathrm{cm})$

IMC $\left(\mathrm{g} / \mathrm{cm}^{2}\right)=$ peso corporal $(\mathrm{g}) /$ altura $2(\mathrm{~cm})$.

Respecto de la grasa corporal, los tejidos adiposos retroperitoneal, epididimal y visceral (mesentérica, perineal, omental) se removieron y pesaron en báscula analítica, y posteriormente se sumaron, revelando el valor total de grasa corporal de cada animal ${ }^{14}$. Además, se computaron el índice de adiposidad (IA) y grasa hepática conforme al método de Folch et al. ${ }^{15}$. El tejido hepático fue homogeneizado y las grasas fueron extraídas con una mezcla de cloroformo y metanol (2:1), seguida de evaporación do solvente en estufa a $105^{\circ} \mathrm{C}$.

IA $(\%)=$ grasa corporal total $(\mathrm{g}) /$ peso corporal

final $(g) \times 100$
Los análisis séricos de glucosa, triglicéridos, colesterol total y fracciones HDL (lipoproteína de alta densidad), LDL (lipoproteína de baja densidad), AST (aspartato aminotransferasa), ALT (alanina aminotransferasa), se realizaron a partir de la sangre colectada en la punción cardíaca, centrifugada a $3000 \mathrm{rpm}$ por 15 minutos. Las evaluaciones fueron realizadas en analizador bioquímico automatizado de la marca Roche ${ }^{\circledR}$ Hitache, Modelo Cobas 6.000, Tokio, Japón, en el Laboratorio de Bioquímica de la UFMS, Campo Grande, MS.

\section{Análisis estadísticos}

Los datos fueron analizados con auxilio del software Statistical Package for the Social Sciences (SPSS), versión 22.0. Todos los datos presentaron distribución normal y fueron sometidos al análisis de variancia (ANOVA), seguido de la prueba post-hoc de Tukey (Tukey's Multiple Comparison Test), siendo considerado significativo $p<0,05$. Los resultados se expresaron como Promedio \pm Error Estándar del Promedio $(\mathrm{P} \pm \mathrm{EEP})$. Letras diferentes en línea de la tabla expresadas en los resultados indican diferencia significativa entre los grupos por la prueba de Tukey $(p<0,05)$. Los resultados de tolerancia a glucosa (PTG), prueba de tolerancia a insulina (PTI) y el subsecuente cálculo de la constante de reducción de la glucosa $\left(\mathrm{K}_{\mathrm{itt}}\right)$ fueron analizados por medio del programa GraphPad Prism, versión 6.0 (GraphPad Software, La Jolla-CA, USA).

\section{RESULTADOS}

La tabla 2 muestra los parámetros antropométricos, grasa corporal, peso del hígado y porcentaje de grasa hepática de los grupos control y experimentales. Se observa que el grupo alimentado con el 6,0\% de polidextrosa presentó significativamente menos IMC $(p=0,003)$, ganancia de peso $(p=0,003)$, circunferencia torácica $(p=0,039)$ y circunferencia abdominal $(p=0,003)$. En cuanto a la grasa corporal $(p<0,001)$, los resultados fueron estadísticamente

Tabla 1. Formulación de las dietas control y experimentales.

\begin{tabular}{|lcccc|}
\hline Ingredientes & $\begin{array}{c}\text { HC } \\
\text { g/Kg dieta }\end{array}$ & $\begin{array}{c}\text { HC2\%P } \\
\text { g/Kg dieta }\end{array}$ & $\begin{array}{c}\text { HC4\%P } \\
\text { g/Kg dieta }\end{array}$ & $\begin{array}{c}\text { HC6\%P } \\
\text { g/Kg dieta }\end{array}$ \\
\hline Ración comercial & 375 & 355 & 335 & 315 \\
Cacahuete tostado & 250 & 250 & 250 & 250 \\
Achocolatado en polvo & 250 & 250 & 250 & 250 \\
Galleta wafer & 125 & 125 & 125 & 125 \\
Polidextrosa (PLX) & - & 20,0 & 40,0 & 60,0 \\
Total & 1.000 & 1.000 & 1.000 & 1.000 \\
\end{tabular}

HC: Dieta Hipercalórica (control); HC2\%P: Dieta hipercalórica con suplementación de 2,0\% del polidextrosa; HC4\%P: Dieta hipercalórica con suplementación del 4,0\% de polidextrosa; HC6\%P: Dieta hipercalórica con suplementación del 6,0\% de polidextrosa.

Fuente: Adaptada de Estadella et al. (2004). Campo Grande (MS), 2018. 
menores en los grupos $\mathrm{HC} \% \mathrm{P}$ y HC6\% $\%$, así como observados en los tejidos adiposos visceral $(p=0,001)$ y epididimal $(p<0,001)$. El tejido adiposo retroperitoneal no se mostró distinto entre los grupos tratados, en cambio en el grupo $\mathrm{HC}$ se presentó significativamente mayor al compararlo con el grupo $\mathrm{HC} 6 \% \mathrm{P}(\mathrm{p}<0,001)$. Los animales alimentados con la polidextrosa $(2,4$ y $6 \%)$ también revelaron menores índices de adiposidad $(p<0,001)$ y grasa hepática $(p<0,001)$ en comparación con los animales del grupo HC (control), destacando que el grupo HC6\%P puntuó una menor acumulación de grasa en el tejido hepático, así como el peso de este órgano (Tabla 2).

En la tabla 3 se pueden observar los resultados de los análisis bioquímicos. Los niveles de glucosa en la sangre fueron estadísticamente más bajos en los grupos $\mathrm{HC} \% \mathrm{P}$ y HC6\%P $(p<0,001)$. En cuanto al colesterol total y la fracción LDL no presentaron diferencias entre los grupos. No obstante, la fracción HDL se reveló significativamente mayor en los animales que consumieron la polidextrosa $(\mathrm{p}=$ $0,001)$, mientras que con relación a los triglicéridos solo el grupo $\mathrm{HC} \% \mathrm{P}$ fue significativamente menor en relación al HC $(p=0,047)$, no habiendo diferencia entre el grupo control y los HC4\%P y HC6\%P. Sobre las actividades de las enzimas hepáticas ALT y AST, la primera no presentó diferencias relevantes. Pero se puede observar un aumento de las actividades enzimáticas AST en los grupos que recibieron dietas con adición de polidextrosa, siendo expresivamente mayor en los grupos HC2\%p Y HC6\%P en relación al $\mathrm{HC}(\mathrm{p}=0,020)$.

En la prueba de tolerancia a glucosa (PTG) no hubo diferencias entre los grupos (Figura $1 \mathrm{~A}$ y B). Respecto a la prueba de tolerancia a la insulina (PTI), el grupo $\mathrm{HC} \% \mathrm{P}$ presento menor resistencia a la insulina, en comparación con los grupos $\mathrm{HC}$ y $\mathrm{HC} 2 \% \mathrm{P}$ (Figura 1C), lo que resultó en un descenso estadísticamente mayor de la tasa de la glucosa (kITT) con relación a los demás grupos (Figura 1D).

Tabla 2. Parámetros antropométricos, grasa corporal, peso del hígado y porcentaje de grasa hepática de los grupos control y experimentales

\begin{tabular}{|c|c|c|c|c|c|}
\hline Parámetros & $\begin{array}{c}\mathrm{HC} \\
\mathrm{P} \pm \mathrm{EEP}\end{array}$ & $\begin{array}{c}\text { HC2\%P } \\
\text { P } \pm \text { EEP }\end{array}$ & $\begin{array}{c}\text { HC4\%P } \\
\text { P } \pm \text { EPP }\end{array}$ & $\begin{array}{c}\text { НС6\% } \% \\
\text { P } \pm \text { EEP }\end{array}$ & $\mathbf{p}$ \\
\hline Peso Inicial (g) & $77,4 \pm 2,70^{\mathrm{ab}}$ & $84,6 \pm 8,00^{a}$ & $79,5 \pm 2,40^{\mathrm{ab}}$ & $63,0 \pm 4,80^{\mathrm{b}}$ & 0,038 \\
\hline Peso Final (g) & $303 \pm 10,0^{\mathrm{a}}$ & $292 \pm 6,84^{\mathrm{ab}}$ & $307 \pm 5,45^{\mathrm{a}}$ & $270 \pm 5,91^{b}$ & 0,003 \\
\hline Ganancia de Peso (g) & $226 \pm 7,99^{a b}$ & $207 \pm 3,32^{\mathrm{bc}}$ & $228 \pm 5,26^{\mathrm{a}}$ & $206 \pm 2,21^{c}$ & 0,003 \\
\hline Largo $(\mathrm{cm})$ & $22,6 \pm 0,229$ & $22,6 \pm 0,300$ & $22,6 \pm 0,158$ & $21,8 \pm 0,188$ & 0,042 \\
\hline *Consumo de la dieta (g) & $14,1 \pm 0,504$ & $14,4 \pm 0,431$ & $15,0 \pm 0,472$ & $13,6 \pm 0,516$ & 0,244 \\
\hline EA $(\%)$ & 5,7 & 5,6 & 5,8 & 5,8 & - \\
\hline $\mathrm{IMC}\left(\mathrm{g} / \mathrm{cm}^{2}\right)$ & $0,590 \pm 0,009^{a b}$ & $0,573 \pm 0,005^{b}$ & $0,604 \pm 0,005^{\mathrm{a}}$ & $0,567 \pm 0,007^{b}$ & 0,004 \\
\hline Índice de Lee $\left(\mathrm{g} / \mathrm{cm}^{3}\right)$ & $0,296 \pm 0,001$ & $0,293 \pm 0,002$ & $0,299 \pm 0,001$ & $0,296 \pm 0,001$ & 0,130 \\
\hline $\mathrm{CT}(\mathrm{cm})$ & $13,9 \pm 0,174^{\mathrm{a}}$ & $13,8 \pm 0,134^{\mathrm{ab}}$ & $13,7 \pm 0,144^{\mathrm{ab}}$ & $13,3 \pm 0,148^{\mathrm{b}}$ & 0,039 \\
\hline $\mathrm{CA}(\mathrm{cm})$ & $16,3 \pm 0,314^{\mathrm{a}}$ & $15,6 \pm 0,271^{\mathrm{ab}}$ & $16,4 \pm 0,151^{\mathrm{a}}$ & $15,2 \pm 0,209^{b}$ & 0,003 \\
\hline Hígado (g) & $10,3 \pm 0,385^{\mathrm{a}}$ & $9,15 \pm 0,333^{b}$ & $10,50 \pm 0,333^{\mathrm{a}}$ & $8,39 \pm 0,333^{b}$ & $<0,001$ \\
\hline Grasa corporal (g) & $14,10 \pm 0,806^{\mathrm{a}}$ & $9,06 \pm 0,698^{b}$ & $12,00 \pm 0,698^{a}$ & $9,04 \pm 0,698^{b}$ & $<0,001$ \\
\hline Visceral (g) & $5,07 \pm 0,313^{a}$ & $3,37 \pm 0,271^{b}$ & $4,40 \pm 0,271^{\mathrm{a}}$ & $3,57 \pm 0,271^{b}$ & 0,001 \\
\hline Epididimal (g) & $4,86 \pm 0,312^{\mathrm{a}}$ & $3,02 \pm 0,215^{b}$ & $4,25 \pm 0,215^{\mathrm{a}}$ & $3,01 \pm 0,215^{b}$ & $<0,001$ \\
\hline Omental (g) & $0,788 \pm 0,072^{\mathrm{a}}$ & $0,461 \pm 0,062^{b}$ & $0,567 \pm 0,062^{b}$ & $0,591 \pm 0,062^{b}$ & 0,018 \\
\hline Perineal (g) & $1,07 \pm 0,095^{\mathrm{a}}$ & $0,593 \pm 0,082^{b}$ & $0,790 \pm 0,082^{b}$ & $0,606 \pm 0,082^{b}$ & 0,002 \\
\hline Mesentérica (g) & $3,21 \pm 0,222^{\mathrm{a}}$ & $2,32 \pm 0,192^{b}$ & $3,04 \pm 0,192^{\mathrm{a}}$ & $2,37 \pm 0,192^{b}$ & 0,006 \\
\hline Retroperitoneal (g) & $4,15 \pm 0,301^{\mathrm{a}}$ & $2,66 \pm 0,260^{\mathrm{ab}}$ & $3,39 \pm 0,260^{\mathrm{ab}}$ & $2,45 \pm 0,260^{\mathrm{b}}$ & $<0,001$ \\
\hline IA (\%) & $4,60 \pm 0,213^{a}$ & $3,09 \pm 0,185^{c}$ & $3,90 \pm 0,185^{b}$ & $3,18 \pm 0,197^{c}$ & $<0,001$ \\
\hline Grasa hepática (\%) & $11,2 \pm 0,339^{a}$ & $8,97 \pm 0,256^{b}$ & $8,52 \pm 0,303^{b}$ & $6,03 \pm 0,256^{c}$ & $<0,001$ \\
\hline
\end{tabular}

Letras diferentes en la línea indican diferencia significativa por la prueba de Tukey ( $<<0,05)$; P: Promedio; EEP: Error Estándar del Promedio; * Promedio de consumo de la dieta por ratas al día; EA: Eficiencia Alimentaria; CT: Circunferencia Torácica; CA: Circunferencia Abdominal; IA: Índice de adiposidad. HC: Grupo con dieta hipercalórica (control); HC2\%: Grupo con dieta hipercalórica + 2,0\% de polidextrosa; HC4\%P: Grupo con dieta hipercalórica $+4,0 \%$ de polidextrosa; HC6\% : Grupo con dieta hipercalórica $+6,0 \%$ de polidextrosa. Fuente: Los autores. Campo Grande (MS), 2018. 
Tabla 3. Parámetros bioquímicos de los grupos control y experimentales.

\begin{tabular}{|lccccc|}
\hline Parámetros & $\begin{array}{c}\text { HC } \\
\mathbf{P} \pm \text { EPP }\end{array}$ & $\begin{array}{c}\text { HC2\%P } \\
\text { P } \pm \text { EPP }\end{array}$ & $\begin{array}{c}\text { HC4\%P } \\
\text { P } \pm \text { EPP }\end{array}$ & $\begin{array}{c}\text { HC6\%P } \\
\text { P } \pm \text { EPP }\end{array}$ & p-valor \\
\hline Glucosa (mg/dL) & $220 \pm 12,9^{\mathrm{a}}$ & $116 \pm 11,2^{\mathrm{b}}$ & $201 \pm 12,9^{\mathrm{a}}$ & $105 \pm 11,9^{\mathrm{b}}$ & $<0,001$ \\
Col. Total (mg/dL) & $69,0 \pm 4,04$ & $74,1 \pm 3,74$ & $71,6 \pm 3,74$ & $75,8 \pm 4,04$ & 0,647 \\
HDL (mg/dL) & $43,3 \pm 2,85^{\mathrm{b}}$ & $54,7 \pm 2,16^{\mathrm{a}}$ & $54,0 \pm 2,16 \underline{\mathrm{a}}$ & $56,0 \pm 2,16^{\mathrm{a}}$ & 0,010 \\
LDL (mg/dL) & $8,48 \pm 1,34$ & $6,70 \pm 1,24$ & $7,75 \pm 1,34$ & $4,81 \pm 1,34$ & 0,268 \\
TGL (mg/dL) & $50,6 \pm 5,31^{\mathrm{a}}$ & $35,9 \pm 4,49^{\mathrm{b}}$ & $53,9 \pm 4.49^{\mathrm{a}}$ & $42,9 \pm 4,49^{\mathrm{ab}}$ & 0,047 \\
AST (U/I) & $121 \pm 20,4^{\mathrm{b}}$ & $198 \pm 17,2^{\mathrm{a}}$ & $151 \pm 17,2^{\mathrm{ab}}$ & $198 \pm 18,6^{\mathrm{a}}$ & 0,020 \\
ALT (U/l) & $43,6 \pm 4,58$ & $49,0 \pm 3,87$ & $50,9 \pm 3,87$ & $53,6 \pm 4,18$ & 0,444 \\
\hline
\end{tabular}

Letras diferentes en línea indican diferencia significativa por la prueba de Tukey $(\mathrm{p}<0,05)$; P: Promedio; EPP: Error Estándar del Promedio; Col. Total: Colesterol Total; HDL: Lipoproteína de Alta Densidad; LDL: Lipoproteína de Baja Densidad; VLDL: Lipoproteína de Muy Baja Densidad; TGL: Triglicéridos; AST: Aspartato Aminotransferasa; ALT: Alanina Aminotransferasa; HC: Grupo con dieta hipercalórica (control); HC2\%P: Grupo con dieta hipercalórica + 2,0\% de polidextrosa; HC4\%P: Grupo con dieta hipercalórica + 4,0\% de polidextrosa; HC6\%P: Grupo con dieta hipercalórica $+6,0 \%$ de polidextrosa.

Fuente: Los autores. Campo Grande (MS), 2018.

A

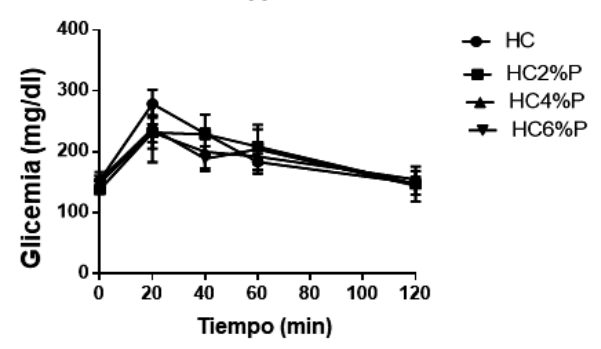

C

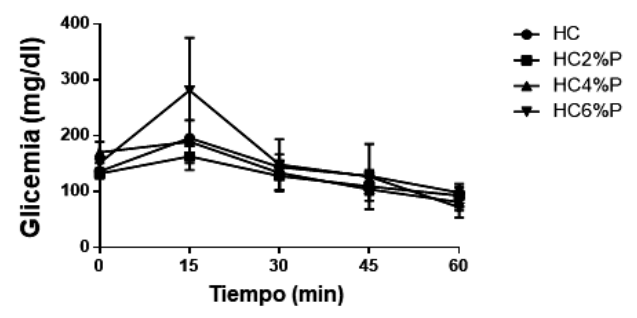

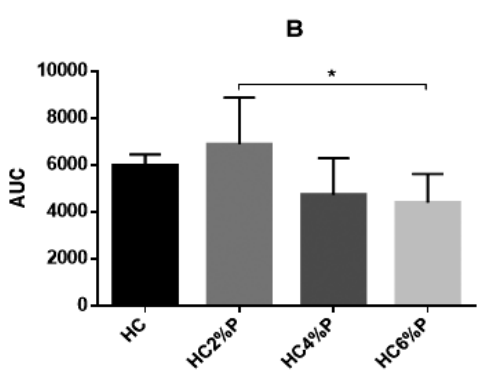

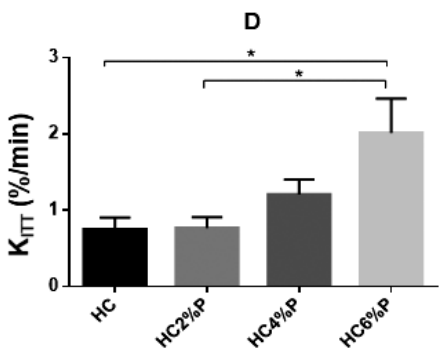

Figura 1: A: Prueba de tolerancia a la glucosa (PTG) aplicada en los tiempos 0, 20, 40, 60 y 120 min; B: área bajo la curva de la prueba de tolerancia a la glucosa (AUC) C: Prueba de tolerancia a insulina (PTI) aplicada en los tiempos 0, 15, 30, 45, 60 min; D: Tasa de descomposición de la glucosa (Kitt) durante la prueba de resistencia a insulina. HC: Grupo con dieta hipercalórica (control); HC2\%P: Grupo con dieta hipercalórica $+2,0 \%$ de polidextrosa; HC4\%P: Grupo con dieta hipercalórica + 4,0\% de polidextrosa; HC6\%: Grupo con dieta hipercalórica + 6,0\% de polidextrosa. Los datos fueron analizados estadísticamente por ANOVA seguido de la prueba post-hoc de Tukey. AUC $=$ Los promedio son significativamente diferentes ${ }^{*} \mathrm{p}<0,05 \mathrm{HC} 2 \% \mathrm{P} \times \mathrm{HC} \% \mathrm{P}$. KITT $=$ Los promedio son significativamente diferentes *p $<0,05 \mathrm{HC} \times \mathrm{HC} \% \mathrm{P}$ e HC2\%P $\times$ HC6\%P.

Fuente: Los autores. Campo Grande (MS), 2018. 


\section{DISCUSIÓN}

Algunas investigaciones sugieren que la polidextrosa, así como la fibra alimenticia, añadida en dietas tiende a aumentar la saciedad ${ }^{9,16}$. No obstante, en el presente estudio, no fue observada la asociación de la fibra en la reducción del apetito, no hubo diferencia en el consumo de la dieta. Este resultado es semejante al estudio de Eufrácio y col que observa que la adición de fibras solubles (pectina y goma guar) en concentraciones del $10,0 \%$ y $15,0 \%$ en dietas hipercolesterolémicas en ratas Wistar ${ }^{17}$ no modifica el consumo de dieta. Pese a que no hay diferencia en la ingesta alimenticia, se observó una menor ganancia de peso en el grupo $\mathrm{HC} \%$ P que en otros grupos Estos datos se distinguen de aquellos encontrados por Pronczuk y Heys ${ }^{18}$, que no encontraron diferencia en la ganancia de peso corporal, en gerbos alimentados con el 6,0\% de polidextrosa, por 4 semanas (tiempo menor que el de este estudio). Sin embargo, estudios con la polidextrosa indican que este nutriente presenta un gran potencial en el control del peso corporal, fundamentando los resultados de este trabajo?.

Una de las asociaciones de la polidextrosa en la regulación del peso corporal son las condiciones de la microbiota intestinal. Según Canfora y Blaak $^{9}$, el consumo de ese prebiótico puede influir en la aparición de bacterias favorables al organismo, como las bifidobacterias y faecalibacterias, siendo las últimas responsables del aumento de la producción de ácidos grasos de la cadena corta (AGCC). Esos AGCC, como el butirato, además de regular la ingesta energética pueden actuar aumentando el gasto energético y la oxidación de grasa, reduciendo el peso corporal, sin alterar el consumo alimentario .

Es válido destacar que la menor ganancia de peso puede justificar los mejores resultados antropométricos IMC, CT y CA del grupo HC6\%P. Resultado similar fue observado por Martins ${ }^{19}$, que verificó la disminución de la ganancia de peso, tendiendo a la reducción del IMC y la CA, así como el porcentaje de grasa corporal en ratas Wistar ovariectomizadas, suplementadas con el $6 \%$ de FOS/inulina del Producto a Base de Yacon (PBY). Además, otros autores también establecen una correlación entre el IMC y la CA con la acumulación de tejido adiposo, señalándolos como indicativos de obesidad en ratas ${ }^{11,20}$.

De acuerdo con los resultados anteriores, fue constatada menor acumulación de grasa corporal, así como en los tejidos adiposos visceral, epididimal y retroperitoneal, en ratas tratados con polidextrosa, principalmente en el grupo $\mathrm{HC} 6 \% \mathrm{P}$, al compararse con el HC. Desenlace similar a uno de los experimentos de Han et al. ${ }^{21}$, que notó menores tejidos adiposos retroperitoneal y epididimal en los animales alimentados con dieta hiperlipídica con adición del 5,0\% de inulina, en comparación a la dieta hiperlipídica con el 5,0\% de celulosa. Así como Raza et al. ${ }^{22}$ que observó menor peso de tejido adiposo epididimal en ratones C57BL/6 sometidos a una dieta occidental suplementada con $75 \mathrm{mg}$ de polidextrosa. Martins ${ }^{19}$, en su estudio, también encontró un menor porcentaje de grasa corporal en animales sometidos a dieta con inulina. Cabe destacar que el índice de adiposidad tiene una vinculación directa con la cantidad de grasa corporal. Este índice también se presentó menor en los grupos que recibieron polidextrosa, siendo un indicativo más de que la polidextrosa evitó la ganancia de masa grasa. Es más, se puede notar que la grasa hepática fue menor en los grupos tratados con polidextrosa.

Una de las justificaciones para el menor valor de grasa corporal de los grupos experimentales $\mathrm{HC} 2 \% \mathrm{P}$ y $\mathrm{HC} \% \% \mathrm{P}$ debe estar relacionada con la disminución del tiempo del tránsito intestinal, retrasando la absorción de carbohidratos y lípidos. Esa lenta absorción de dichos nutrientes puede disminuir la glucosa postprandial y la sensibilidad a la insulina que posiblemente induce a la reducción de la inhibición de la lipólisis y el aumento de la oxidación de lípidos, reduciendo la acumulación de grasa corporal9.

En el presente estudio no se observó el efecto de la polidextrosa sobre el colesterol total y el LDL, pero se detectaron niveles elevados del HDL en los grupos suplementados. Ese resultado está de acuerdo con los trabajos de Kim y Shin ${ }^{23}$, que tampoco detectaron efectos de las fibras solubles (del extracto de la achicoria y de los granos de amaranto y linaza, respectivamente) en el colesterol total, pero constataron un aumento en los niveles del colesterol HDL en los grupos tratados. En cambio, algunos estudios muestran que la polidextrosa tiene un gran potencial en la reducción de los niveles del colesterol total y triglicéridos ${ }^{18,24}$. Cicek et al. ${ }^{24}$ observaron que el consumo de la polidextrosa y de la oligofructosa en pacientes con diabetes tipo 2 redujo los niveles séricos del colesterol total, LDL y triglicéridos, además de aumentar los niveles de HDL. En gerbos, también se encontraron menores niveles de colesterol total y no-HDL (LDL, VLDL, IDL), no siendo observado el efecto de la polidextrosa en los niveles de HDL en cuatro semanas, tiempo menor de experimento, si se compara con el presente estudio ${ }^{18}$.

Respecto a los triglicéridos, el grupo HC2\%P se presentó estadísticamente menor en comparación al grupo $\mathrm{HC}$, resultado semejante a los estudios con el 5,0\% de inulina ${ }^{21}$ y $75 \mathrm{mg}$ de polydextrosa ${ }^{22}$. No obstante, los demás grupos tratados con la polidextrosa ( $\mathrm{HC} 4 \% \mathrm{P}$ y HC6\%P) no tuvieron diferencia en relación al control, coincidiendo con el estudio de Pronczuk y Heys ${ }^{18}$. A pesar de conflictivo, se sabe que las fibras solubles tienen efectos en el control de los triglicéridos, además de la importante característica hipocolesterolémica, como la reducción del colesterol total y del LDL, y el aumento del HDL, siendo factores importantes en el control de las dislipidemias ${ }^{25,26}$. Hay muchas explicaciones a respecto del efecto hipocolesterolémico de las fibras. Una de ellas es que las fibras se unen a los ácidos biliares aumentando su excreción y disminuyendo el colesterol ${ }^{25,26}$. Otra justificación es la disminución del tiempo del tránsito intestinal, que según los autores esa lentitud disminuyó la absorción de los nutrientes, entre ellos la absorción de grasas, contribuyendo a la reducción sérica de los triglicéridos ${ }^{9,26}$. Además, Surampudi et al. ${ }^{26}$ explica que los AGCC, como el acetato y el propionato, producidos por las fermentaciones de los prebióticos, tienen 
funciones hipolipemiantes. Los AGCC, tras ser producidos, actúan directamente en el hígado, inhibiendo el metabolismo del colesterol y la lipogénesis. Cabe resaltar que algunos estudios mencionan que el contenido de las bifidobacterias tiene una relación positiva con el aumento del HDL y que la polidextrosa tiene como efecto en el organismo la promoción del crecimiento de esas bacterias, 727,28 . Si bien el actual estudio no ha encontrado efectos de la polidextrosa en la reducción del colesterol total y LDL, los niveles altos de HDL ya son un importante factor protector para el riesgo de enfermedades cardiovasculares ${ }^{27,29,30}$.

En el análisis de los parámetros bioquímicos, se investigaron, asimismo, las actividades enzimáticas. En el presente trabajo se observó que los animales que recibieron la añadidura de la polidextrosa en la dieta presentaron mayores niveles de actividad AST, principalmente en los grupos $\mathrm{HC} 2 \% \mathrm{P}$ y $\mathrm{HC} \% \% \mathrm{P}$, comparados con el HC. Estas enzimas son consideradas importantes marcadores de disfunción hepática ${ }^{31}$. Sin embargo, no se puede afirmar que los niveles altos de AST sean un indicativo de lesión hepática, una vez que esta enzima no es un indicador específico del daño hepático, ya que también se encuentra en otros tejidos como el corazón, el músculo esquelético, riñones, páncreas y cerebro $^{32}$. Esta enzima también puede presentarse más elevada por la influencia de anestesia y estrés muscular, a los que se someten las ratas para la colecta de sangre ${ }^{33}$. Además, en este estudio no se observó cambio alguno sobre el ALT, un parámetro más sensible para la hepatotoxicidad que el AST, puesto que es encontrada fundamentalmente en el hígado ${ }^{32}$. Al considerarse valores de referencia de Rattus novergicus linaje Wistar, ambas enzimas AST y ALT presentaron valores cercanos a los considerados normales para ratas ${ }^{33}$.

Respecto al metabolismo glucémico, es un hecho conocido que las fibras solubles prebióticas tienen grandes efectos beneficiosos, como el control del azúcar en la sangre ${ }^{34,35}$. En investigación hecha con harina de la cáscara del maracuyá (fuente de fibra soluble) se mostró que la dieta con el 5,0\% de esa harina redujo los niveles de glucosa plasmática en un $59,0 \%$ en ratas diabéticas ${ }^{34}$. En humanos se observó que la ingesta de polidextrosa por individuos diabéticos disminuyó la glicemia de ayuno y la postprandia ${ }^{24}$. Esos efectos fueron también verificados en las dosificaciones de la glucosa sérica del actual estudio, mostrando que los animales de los grupos HC2\%P y HC6\%P presentaron menores tasas de azúcar en la sangre. Además, la polidextrosa tiene un índice glucémico muy bajo ${ }^{7,27}$, siendo también una de las explicaciones del menor contenido de glucosa sanguínea encontrado en el grupo suplementado con el 6,0\% de polidextrosa.

En la prueba de tolerancia a la glucosa, el grupo suplementado con el mayor porcentaje de polidextrosa ( $\mathrm{HC} \%$ P) fue el que presentó la menor tolerancia a glucosa, con relación al grupo que recibió menor cantidad de ese prebiótico, el HC2\%P. Además, el grupo HC6\%P presentó mayor tasa de reducción de la glucosa en comparación con el grupo control HC, mostrándose una menor resistencia a insulina. El índice KITT corresponde a la caída de la glucosa expresada en \%/minuto. Cuanto más rápida e intensa es la caída de la glucosa (KITT), mayor será la sensibilidad a la insulina ${ }^{36}$. Coincidiendo con los resultados del actual estudio, investigaciones verificaron que los individuos tratados con prebióticos eran menos intolerantes a la glucosa ${ }^{9}$. Una investigación con ratas, mostró que los grupos tratados con la inulina tuvieron menor AUC de la prueba de tolerancia a la glucosa en relación al control ${ }^{21}$. Fue verificado lo mismo en el estudio con polidextrosa en humanos, en el que el AUC de la glucosa fue menor en los grupos que recibieron mayores concentraciones de ese prebiótico en la dieta7.

Se sabe que la polidextrosa tiene posibles efectos en el metabolismo de la glucosa, disminuyendo los picos de respuestas glucémicas y de insulina postprandiales y mejorando el control metabólico glucémico de pacientes diabéticos ${ }^{7,9,27}$. Una de las fundamentaciones para tales efectos glucémicos es la reducción de la absorción excesiva de glucosa en el intestino, a causa de la disminución del tiempo del tránsito intestinal, retrasando el transporte y mezclas de enzimas en el quimo, debido a la característica de fibra soluble de la polidextrosa9, ${ }^{9}$. La mejora de la sensibilidad de la insulina también puede estar asociada a los AGCC, particularmente el acetato y el butirato, producidos por la fermentación microbiana de la polidextrosa. Esos AGCC aumentan las concentraciones de hormonas reguladoras de la saciedad como péptido YY (PYY) y Glucagon-like Peptideo 1 (GPL1), que actúan inhibiendo la producción de glucagón, aumentando la secreción de insulina inducida por la glucosa y mejorando la sensibilidad a la insulina9 .

\section{CONCLUSIÓN}

En conclusión, el consumo de la polidextrosa, principalmente en la concentración del 6,0\%, ejerce un gran papel en el control de la obesidad y dislipidemias, aun en una dieta hipercalórica. Los animales que recibieron esa suplementación presentaron menor ganancia de peso corporal, y en consecuencia, menores valores para el IMC, la CT y la CA, además de menor acumulación de tejido adiposo. Inversamente, dicho grupo presentó mayores valores para el HDL. Sin embargo, cabe resaltar que el grupo con la suplementación del 2,0\% de polidextrosa presentó menores niveles de triglicéridos.

Además, fue notada la relevancia de la polidextrosa en la regulación de la glicemia, pues los grupos que recibieron mayor cantidad de ese prebiótico tuvieron menores tasas de azúcar en la sangre, baja intolerancia a la glucosa y resistencia a la insulina. Así que esos datos pueden ser utilizados para el aumento del conocimiento y generar nuevas estrategias para la prevención/tratamiento de la obesidad y sus complicaciones, como la diabetes tipo II y las enfermedades cardiovasculares.

\section{BIBLIOGRAFÍA}

1. Hruby A, Hu FB. The Epidemiology of obesity: A big picture. Pharmacoeconomics 2015; 33(7): 673-689.

2. World Health Organization [Homep age en internet]. Obesity 
and overweight [acesso en 10/09/2019]. Disponible en: < http://www.who.int/en/news-room/fact-sheets/detail/obesityand-overweight/>

3. Suárez-Carmona, W, Sánchez-Oliver, AJ, González-Jurado, JA. Pathophysiology of obesity: Current view. Rev Chil Nutr 2017; 44(3): 226-233.

4. Smethers AD, Rolls BJ. Dietary management of obesity: cornerstones of healthy eating patterns. Med Clin North Am 2018; 102(1): 107-124.

5. Davani-Davari D, Negahdaripour M, Karimzadeh I, Seifan $M$ et al. Prebiotics: Definition, Types, Sources, Mechanisms, and Clinical Applications. Foods 2019; 8(3): 92.

6. Khangwal I, Shukla P. Potential prebiotics and their transmission mechanisms: Recent approaches. J. Food Drug Anal 2019; 27: 649-656.

7. Jie Z, Bang-Yao L, Ming-Jie X, Hai-Wei L. Zu-Kang Z, Ting-Song $W$, Craig SA. Study on the effects of polydextrose intake on physiologic functions in Chinese. Am J Clin Nutr 2000; 72: 1503-1509.

8. Carmo MMR, Walker JCL, Novello D, Caselato VM et al. Polydextrose: Physiological Function, and Effects on Health. Nutrients 2016; 8(9): 553.

9. Canfora EE, Blaak EE. The role of polidextrose in body weight control and glucose regulation. Curr Opin Clin Nutr Metab Care 2015; 18: 395-400.

10. Estadella D, Oyama LM, Dâmaso AR, Ribeiro EB, Nascimento CMO. Effect of Palatable Hyperlipidic Diet on Lipid Metabolism of Sedentary and Exercised Rats. Nutr 2004; 20: 218-224.

11. Novelli ELB, Diniz YS, Galhardi CM, Ebaid GMX, Rodrigues HG, Mani F, et al. Anthropometrical parameters and markers of obesity in rats. Lab Anim 2007; 41:111-119.

12. Lenquiste SA, Batista AG, Marineli RS, Dragano NRV, Maróstica Jr MR. Freeze-dried jaboticaba peel added to high-fat diet increases HDL-cholesterol and improves insulin resistance in obese rats. Food Res Int 2012; 49: 153-160.

13. Costa Jr JM, Ferreira SM, Protzek AO, Santos GJ, Cappelli AP, Silveira $L R$, et al. Endurance Training Inhibits Insulin Clearance and IDE Expression in Swiss Mice. PloS One 2015; 10(3): 1-14.

14. Nascimento OV, Boleti APA, Yuyama LKO, Lima ES. Effects of diet supplementation with Camu-camu (Myrciaria dúbia HBK MCVaugh) fruit in a rat model of diet-induced obesity. An Acad Bras Ciênc 2013; 85(1): 355-363.

15. Folch J, Lees M, Sloane SGH. A simple method for the isolation and purification of total lipides from animal tissues. I Biol Chem 1957; 226(1): 497-509.

16. Ibarra A, Astbury NM, Olli K, Alhoniemi E, Tiihonen K. Effects of polydextrose on diferente levels of energy intake. A systematic review and meta-analysis. Appet 2015; 87: 30-37.

17. Eufrásio MR, Barcelos MFP, Souza RV, Abreu WC, Lima MAC, Pereira MCA. Effect of different types of fibers on the lipid portions of wistar rat blood and liver. Ciênc Agrotec Lavras 2009; 33(6): 1608-1614.

18. Pronczuk a, Hayes KC. Hypocholesterolemic effect of dietary polydextrose in gerbils and humans. Nutr Res 2006; 26: 2731.

19. Martins JFL, Rafael VC, Filomena EA, Rodrigues FC, Sartori SSCR, Ferreira CLLF. Product based yacon and modulation of microbiota, fatty acid profile and lipid in ovariectomized female rats. Rev Nutr 2016; 29(5): 709-719.

20. Angeloco $L R N$, Deminice $R$, Leme IA, Lataro $R C$, Jordão $A A$.
Bioelectrical impedance analysis and anthropometry for the determination of body composition in rats: effects of high-fat and high-sucrose diets. Rev Nutr 2012; 25(3):331-339.

21. Han KH, Tsuchihira H, Nakamura Y, Shimada K, Ohba K, Aritsuka T.Inulin-type fructans with different degrees of polymerization improve lipid metabolism but not glucose metabolism in rats fed a high-fat diet under energy restriction. Dig Dis Sci 2013; 58(8): 2177-2186.

22. Raza GS, Putaala H, Hibberd AA, Alhoniemi E. Polydextrose changes the gut microbiome and attenuates fasting triglyceride and cholesterol levels in Western diet fed mice. Sci Rep 2017; 7.

23. Kim M, Shin HK. The water-soluble extract of chicory influences serum and liver lipid concentrations, cecal short- chain fatty acid concentrations and fecal lipid xxcretion in rats. I Nutr 1998; 128(10): 1731-1736.

24. Cicek B, Arslan P, Kelestimur F. The Effects of oligofructose and polidextrose on metabolic control parameters in type- 2 diabetes. Park J Med Sci 2009; 25(4): 573-578.

25. Sima P, Vannucci L, Vetvicka V. $\beta$-glucans and cholesterol. Int J Mol Med 2018; 41: 1799-1808.

26. Surampudi P, Enkhmaa B, Anuurad E, Berglund L. Lipid Lowering with Soluble Dietary Fiber. Curr Atheroscler Rep 2016; 18(75).

27. Putaala H. Polydextrose in Lipid Metabolism. Lip Metab, [Internet]. 2013. DOI: 10.5772/51791. Disponible em <http:// www.intechopen.com/books/lipid-metabolism/polydextrosein-lipid-metabolism>.

28. Ichim TE, Patel AN, Shafer KA. Experimental support for the effects of a probiotic/digestive enzyme supplement on serum cholesterol concentrations and the intestinal microbiome. I Transl Med 2016; 14(184).

29. Field FJ, Watt K, Mathur SN. Origins of intestinal ABCA1mediated HDL-cholesterol. I Lip Res 2008; 49: 2605-2619.

30. Nicholls SJ, Nelson AJ. HDL and vascular desease. Pathology 2019; 51(2): 142-147.

31. Jesus GC, Sousa HHBA, Barcelos RSS. Major adverse change of liver and biomarkers. Estudos 2014; 41(3): 525-537.

32. Reis TA, Goulart PF, Oliveira RME, Oliveira L, Abreu OS, Azevedo AO. Metabolic parameters of wistar rats subjected to diet supplemented with stevia and sugar. Semina: Ciênc Agr 2011; 32(4): 1477-1488.

33. Melo MGD, Dória GAA, Serafini MR, Araújo AAS. Reference values of hematological and biochemical rats (Rattus norvegicus Wistar strain) from the central animal house of the Federal University of Sergipe. Sci Plena 2012; 8(9): 1-6.

34. Salgado JM, Bombarde TAD, Mansi DN, Piedade SMS, Meletti LMM. Effects of different concentrations of passion fruit peel (Passiflora edulis) on the glicemic control in diabetic rat. Ciênc Tecnol Aliment 2010; 30(3): 784-789.

35. Lightowler H,Thondre S, Holz A, Theis S. Replacement of glycaemic carbohydrates by inulin-type fructans from chicory (oligofructose, inulin) reduces the postprandial blood glucose and insulin response to foods: report of two doubleblind, randomized, controlled trials. Eur J Nutr 2018; 57(3): 12591268.

36. Abdul-ghani MA, Matsuda M, Balas B, DeFronzo RA. Muscle and liver insulin resistance indexes derived from the oral glucose tolerance test. Diabetes Care, New York 2007; 30(1): 89-94. 